\title{
Performance of Sorting Algorithms on the SRC 6 Reconfigurable Computer
}

\author{
John Harkins, Tarek El-Ghazawi, Esam El-Araby, Miaoqing Huang \\ The George Washington University \\ johnharkins@ieee.org, \{tarek,esam,mqhuang\}@gwu.edu
}

\begin{abstract}
The execution speed of the FPGA processing elements are compared to the microprocessor processing elements in the SRC 6 reconfigurable computer using the following algorithms for sorting: Quick Sort, Heap Sort, Radix Sort, Bitonic Sort, and Odd/Even Merge. The results show that, for sorting, FPGA technology may not be the best processor choice and that factors such as memory bandwidth, clock speed, algorithm computational density and an algorithm's ability to be pipelined all have an impact on FPGA performance.
\end{abstract}

Keywords: reconfigurable computer, sorting, FPGA, hardware compiler, SRC, Quick Sort, Heap Sort, Radix Sort, Bitonic Sort, Odd/Even Merge.

\section{Introduction}

Field Programmable Gate Array (FPGA) technology has recently been added to several highperformance computing systems as a means to increase overall system performance ([1], [2], [3]). In this study, we use a reconfigurable computer from SRC Computers to evaluate the performance of reconfigurable computers for sorting.

\section{Hardware Implementations}

Quick Sort (QS) [4] and Heap Sort (HS) [5] are implemented as six multiple instruction multiple data (MIMD) [6] instances operating in parallel; the QS instances are arranged as three per two FPGAs and the HS instances are six per one FPGA. Radix Sort (RS) [7] is implemented as three MIMD instances in a single FPGA. Bitonic Sort (BS) [8] is implemented as two single instruction multiple data (SIMD) [6] instances; the first is an eight input parallel sorting network virtualized [9] to $2^{21}$ inputs and the second is a four input parallel sorting network virtualized to $2^{20}$ inputs. Finally, the Odd/Even Merge (OE) hardware is a parallel network that merges two sorted vectors into one. All codes except BS were written using SRC's C to gates compiler; the BS implementation was written using VHDL. All sorts except for RS and OE are done inplace.
Prior to measuring execution speeds, we derive and compute an upper bound on the performance for each hardware implementation (see column 4 in Table 1).

\section{Test Conditions}

The benchmark scores compare a single microprocessor node to a single FPGA node in the SRC 6. Microprocessor codes were written in $\mathrm{C}$ and were not designed to emulate the hardware implementations but rather were chosen to be efficient for sequential processors. Execution times were measured using the $\mathrm{C}$ function gettimeofday(). Equal key quantities were used on the microprocessors and the FPGAs for a given algorithm.

Keys were uniformly distributed and randomly permuted prior to each iteration and the reported speeds are the average for each sort over ten consecutive iterations. The average for the FPGAs includes the configuration time (that occurs on only the first of the ten iterations for each sort function) and the time required to DMA the keys into and out of the local FPGA memories. The key size and type for all benchmarks was 64 bit unsigned integers.

\section{Experimental Results}

The results shown in Table 1 indicate that the RS implementation in a single FPGA was the overall winner, with a slightly greater than $2 \mathrm{x}$ improvement over QS on the processor. Overall, the results for the FPGAs were not as strong as anticipated and the microprocessor results were stronger than expected.

In the case of QS and HS, we notice that our actual performance is more than $10 \mathrm{x}$ below what we computed for the upper bound. This is mainly due to dependencies that prevented the SRC $\mathrm{C}$ to gates compiler from fully pipelining the hardware. On the other hand, with RS, the compiler performed much better only adding as few as two clock penalties to the RS pipeline. The BS implementation is fully pipelined but must be slowed down by five clocks due to the memory resource conflict created by sorting in-place. Finally, after the addition of hardware prediction circuitry, OE clock penalties were reduced to one for a top speed of half of its 
Table 1 Sorting performance for 64 bit keys.

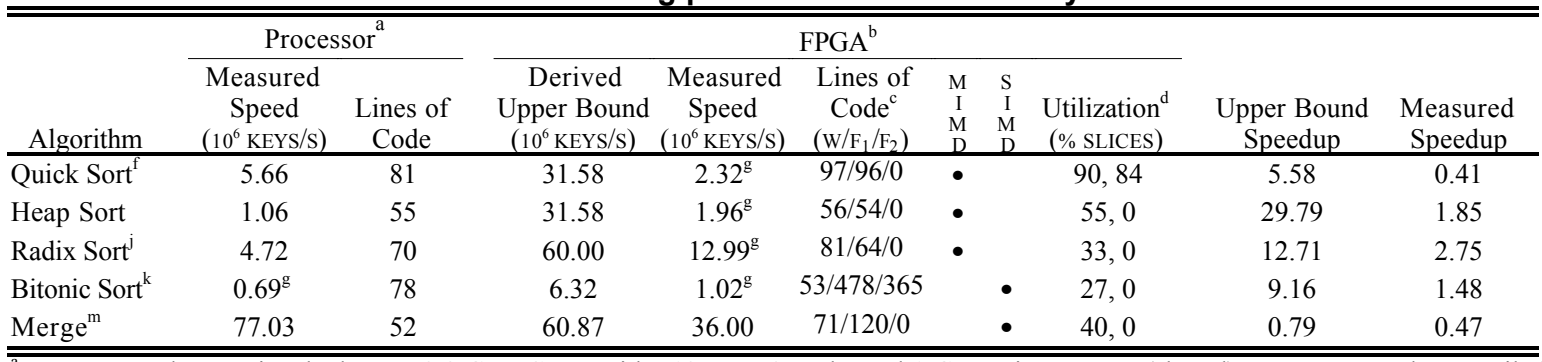

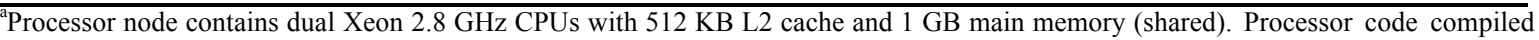
with icc version 8.0 using -fast optimization switch.

${ }^{\mathrm{b}}$ FPGA nodes contain dual Xilinx VertexII6000 FPGAs clocked at $100 \mathrm{MHz}$ with $24 \mathrm{MB}$ local memory (shared). FPGA functions compiled using mcc version 1.8 except where noted.

${ }^{\mathrm{c}}\left(\mathrm{W} / \mathrm{F}_{1} / \mathrm{F}_{2}\right)$ where $\mathrm{W}$ is structural wrapper (not germane to algorithm) and $\mathrm{F}_{1} \& \mathrm{~F}_{2}$ are the sort macros or inlined functions

${ }^{\mathrm{d}}$ SRC overhead $\sim 5 \% .0$ indicates $2^{\text {nd }}$ FPGA not used.

${ }^{\mathrm{f}}$ Microprocssor code is recursive \& uses Insertion Sort for partitions $\leq 20$; FPGA code is non-recursive \& uses a pipeline sorter similar to Bubble Sort for partitions $\leq 10$.

${ }^{\mathrm{g}}$ Results not fully merged.

${ }^{\mathrm{j}}$ Radix for microprocessor is 256; Radix for FPGA is 8192 .

${ }^{\mathrm{k}}$ Microprocessor code is recursive; FPGA uses bitonic sorting network implemented in VHDL compiled using mcc version 1.9.

${ }^{\mathrm{m}}$ Processor uses two-way merge; FPGA uses Odd/Even Merge compiled using mcc v1.9.

computed upper bound.

The utilization results indicate that the SRC 6 may not be optimally balanced for the task of sorting. QS was the only implementation to utilize more than one FPGA and in all other sorts, even the single FPGA went underutilized. Doubling the number of parallel memory channels available to the FPGAs from 6 to 12 will approximately double the performance of those sorts that only utilized one FPGA. Furthermore, a second doubling from 12 to 24 memory channels should allow full utilization of the slices available to each FPGA and again double the performance of those sorts with utilization below $50 \%$. (Note: although memory bandwidth can also be improved with the use of FPGA block RAMs, we did not use them due to their relatively small size).

\section{Conclusion}

Our results indicate that FPGAs may not be the best choice for the task of sorting. We might expect approximately a $4 \mathrm{x}$ improvement over the results we achieved if the architectural improvements mentioned in the previous section are made. Of course, there may be opportunity for additional gains through alternate algorithms or further re-factoring of the presented algorithms.

Because significantly better results using the SRC 6 against other problems ([10], [11], [12]) have been demonstrated, further study is needed to find the characteristics that determine a problems suitability to acceleration with FPGAs. One difference between our study and previous studies is the low computational density per memory access required for sorting; sorting is largely a memory bound process. This may explain why our performance and FPGA utilization was low. Conversely, it may also explain why microprocessors are very good for like problems. At close to 30x slower than the microprocessor, the FPGA must make up that clock speed difference by doing much more work per clock cycle. However sorting capacity is largely governed by memory bandwidth that, in this case, limits performance.

\section{References}

[1] D. S. Poznanovic, "Application Development on the SRC Computers, Inc. Systems", Proceedings of the $19^{\text {th }}$ IEEE International Parallel and Distributed Processing Symposium, IPDPS 2005.

[2] Silicon Graphics. (2004, November). "Extraordinary Acceleration of Workflows with Reconfigurable Application Specific Computing" [Online]. Available: http://www.sgi.com/pdfs/3721.pdf

[3] D.H. Brown Associates. (2004, October). "Cray XD1 Brings High-Bandwidth Supercomputing to the MidMarket" [Online]. Available: http://www.cray.com/downloads/dhbrown_crayxd1_oct 2004.pdf

[4] T. H. Cormen, C. E. Leiserson, R. L. Rivest, C. Stein, Introduction to Algorithms, $2^{\text {nd }}$ ed., The MIT Press, Cambridge, Mass., May 2001, Chap. 7.

[5] Cormen et al., Introduction to Algorithms, Chap. 6.

[6] Selim G. Akl, Parallel Sorting Algorithms, Academic Press, Orlando, Florida, 1985, pp. 4-7.

[7] Cormen et al., Intro. to Algorithms, pp. 168-173

[8] Akl, Parallel Sorting Algorithms, pp. 29-37,65-75.

[9] S. Olariu, M. Christina Pinotti, S. Q. Zheng, "How to Sort N Items Using a Sorting Network of Fixed I/O Size", IEEE Transactions on Parallel and Distributed Systems, May 1999, Vol. 10, No. 5, pp. 487-499.

[10]S. Bajracharya, C. Shu, K. Gaj, T. El-Ghazawi, "Implementation of Elliptic Curve Cryptosystems over $\mathrm{GF}\left(2^{\mathrm{n}}\right)$ in Optimal Normal Basis on a Reconfigurable Computer", $14^{\text {th }}$ International Conference on Field Programmable Logic and Applications, FPL 2004, Antwerp, Belgium, Sept. 2004, pp 1001-1005.

[11]E. El-Araby, T. El-Ghazawi, J. Le Moigne, K. Gaj, "Wavelet Spectral Dimension Reduction of Hyperspectral Imagery on a Reconfigurable Computer", Field Programmable Technology, FPT 2004, Brisbane, Australia, Dec. 2004.

[12] O. D. Fidanci, D. Poznanovic, K. Gaj, T. El-Ghazawi, N. Alexandritis, "Performance Overhead in a Hybrid Reconfigurable Computer", Reconfigurable Arch. Workshop, RAW 2003, Nice, France Apr. 2003. 